\title{
Tunable Channel Drop Filter in a Two-Dimensional Photonic Crystal Modulated by a Nematic Liquid Crystal
}

\author{
Chen-Yang Liu and Lien-Wen Chen \\ Department of Mechanical Engineering, National Cheng Kung University, No.1, Ta-Hsueh Road, Tainan 701, Taiwan
}

Received 27 September 2005; Revised 15 February 2006; Accepted 23 February 2006

Recommended for Publication by Bohua Sun

Photonic crystals (PCs) have many potential applications because of their ability to control light-wave propagation and because PC-based waveguides may be integrated into optical circuits. We propose a novel tunable PC channel drop filter based on nematic liquid crystals and investigate its properties numerically by using the finite-difference time-domain (FDTD) method. The refractive indices of liquid crystals can be actively modulated after infiltrating nematic liquid crystals into the microcavity in PC waveguides with square lattices. Then we can control light propagation in a PC waveguide. We analyze the Q-factors and resonance frequencies of a tunable PC channel drop filter by considering various indices modulation of liquid crystals. The novel component can be used as wavelength division multiplexing in photonic integrated circuits.

Copyright (c) 2006 C.-Y. Liu and L.-W. Chen. This is an open access article distributed under the Creative Commons Attribution License, which permits unrestricted use, distribution, and reproduction in any medium, provided the original work is properly cited.

\section{INTRODUCTION}

Photonic crystals (PCs) are artificial dielectric or metallic structures in which the refractive index modulation gives rise to stop bands for optical waves within a certain frequency $[1,2]$. The waveguide creates a band of conduction inside the bandgaps. These crystals have many potential applications because of their ability to control light-wave propagation and because PC-based optical waveguides may be integrated into optical circuits. The periodicity is broken by introduction of some defects into the crystals. It has been shown that doped PCs permit the guiding of waves in two different geometric paths for two distinct wavelength ranges [3]. Such structures can be used to design highly efficient new optical devices. Optical waveguides in two-dimensional (2D) PCs produced by insertion of linear defects into PC structures had been proposed [4] and experimentally proved [5]. PCs had attracted much attention in the fabrication of high$Q$ microcavities. The introduction of a local defect inside a perfect 2D periodic dielectric structure may give rise to a sharp resonant state inside the crystal in the vicinity of the defect. Villeneuve et al. investigated the properties of a tunable single-mode waveguide microcavity that is well studied for frequency modulation and switching $[6,7]$. Planar PC circuits consist of devices, such as splitters [8], filters [9], and multichannel drop filters [10], by controlling the interaction between static devices, such as waveguides, cavities, or horns. However, for many wavelength division multiplexing (WDM) applications, it would prove advantageous to tune these devices to some degree to enable wavelength selectivity. A multichannel WDM system consisting of a 2D photonic crystal was proposed [11]. The system consists of two parts, a waveguiding element, realized by defects in a photonic crystal, and frequency-selective elements, realized by photonic crystal microcavities. Simulations showed the ability to filter an incident pulse into six spectral channels with an FWHM of $2 \mathrm{~nm}$.

It is important, however, to obtain tunable PC waveguides for applications in optical devices. Busch and John demonstrated that when an optically birefringent nematic liquid crystal is infiltrated into the void regions of an inverse opal, photonic bandgap material, the resulting composite material exhibits a completely tunable photonic bandgap [12]. Tunable PC waveguides that utilize synthetic opals and inverse opals infiltrated with functional materials have been proposed $[13,14]$. One can control the refractive indices of opals by adjusting various factors and fields. For example, one can change the refractive indices of conducting polymers and liquid crystals (LCs) by changing the temperature and the electric field of the polymer or crystal. Therefore one can 


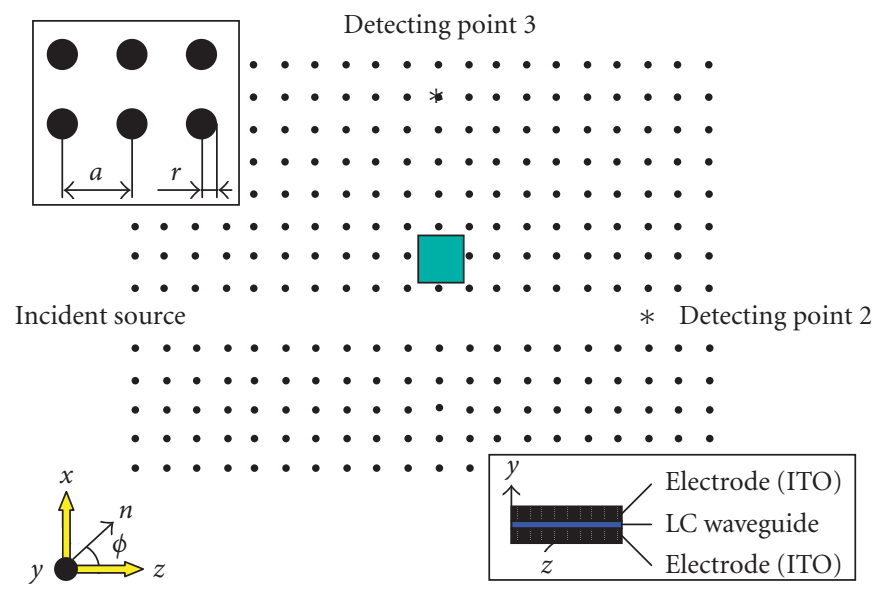

FIGURE 1: PC channel drop filter design for a square lattice and the shaded region are parts infiltrated with liquid crystals. The diagram in lower left corner indicates the director of a liquid crystal. The left inset shows the lattice constant and the radius of rods. The right inset shows the electrode configuration.

change the optical properties of tunable PC waveguides composed of such materials by changing the temperature and the electric field in the same way. Recently the propagation of tunable light in $Y$-shaped waveguides in 2D PCs by use of LCs as linear defects was proposed $[15,16]$. The tunable PC waveguide coupler based on nematic LCs was presented by the authors [17].

In the present report, a novel tunable PC waveguide channel drop filter with nematic liquid crystals is proposed, and its properties are numerically investigated by using the finite-difference time-domain (FDTD) method. The PC channel drop filter can be obtained by the infiltration of LCs into the microcavity in 2D PC waveguides with square lattices. The resonant modes can be changed in microcavity based on the orientation of LCs by adjusting the applied field. It makes controlling the lightwave with a certain frequency in $\mathrm{PC}$ waveguide possible. We incorporate these tunable cavities in a channel drop filter to demonstrate their enhancement of PC-WDM applications.

\section{FINITE-DIFFERENCE TIME-DOMAIN APPROACH}

The finite-difference time-domain method was introduced by Kane Yee in 1966 firstly. The approach is based on a direct numerical solution of the time-dependent Maxwell's equations by use of the finite-difference technique [18]. We treat the case of the TE mode and directors of LCs parallel to 2D planes, because the electric field exists only in the 2D planes in this mode. The electric field is strongly affected by rotating directors of LCs [15]. To determine the dispersion relations of guided modes in PC waveguides with nematic LCs we can express the light-wave equation that is satisfied by the magnetic field for $2 \mathrm{D}$ planes as

$$
\nabla \times\left[\frac{1}{\varepsilon(r)} \nabla \times H(r)\right]=\left(\frac{\omega}{c}\right)^{2} H(r)
$$

where the dielectric tensor $\varepsilon(r)=\varepsilon(r+R)$ is periodic with respect to lattice vector $R$ generated by primitive translation, and $\nabla \cdot H(r)=0$.

Generally LCs possess two kinds of dielectric indces. One is ordinary dielectric index $\varepsilon^{o}$, and the other is extraordinary dielectric index $\varepsilon^{e}$. Light waves with electric fields perpendicular and parallel to the director of the LC have ordinary and extraordinary refractive indices, respectively. In a 2D plane, the components of the dielectric tensor of the nematic LC are represented as [19]

$$
\begin{gathered}
\varepsilon_{x x}(r)=\varepsilon^{o}(r) \sin ^{2} \phi+\varepsilon^{e}(r) \cos ^{2} \phi, \\
\varepsilon_{z z}(r)=\varepsilon^{o}(r) \cos ^{2} \phi+\varepsilon^{e}(r) \sin ^{2} \phi, \\
\varepsilon_{x z}(r)=\varepsilon_{z x}(r)=\left\lfloor\varepsilon^{e}(r)-\varepsilon^{o}(r)\right\rfloor \cos \phi \sin \phi,
\end{gathered}
$$

where $\phi$ is the rotation angle of the director of the LCs, and $n=(\cos \phi, \sin \phi)$ is the director of the LC, as shown in Figure 1.

The FDTD method is used to solve the light propagation in 2D PC waveguide with LCs. The FDTD algorithm must be modified at the boundaries of the computational region where suitable numerical absorbing boundary condition are applied. The perfectly matched layer boundary condition [20] has been used because it yields high performance. The photonic device is laid out in the $x-z$ plane. The propagation is along $z$ direction. The space steps in the $x$ and $z$ direction are $\Delta x$ and $\Delta z$, respectively. Yee's numerical scheme applied to the 2D TE case. It uses central difference approximations for the numerical derivatives in space and time, both having second-order accuracy. The sampling in space is on a subwavelength scale. Typically, 10 to 20 steps per wavelength are needed. We assume that $\Delta x=0.07$ and $\Delta z=0.07$. The sampling in time is selected to ensure numerical stability of the algorithm. The time step is determined by the Courant limit [18]. A more-detailed treatment of the FDTD method is given in [18]. 


\section{TUNABLE PHOTONIC CRYSTAL CHANNEL DROP FILTER}

The novel tunable PC channel drop filter composed of dielectric pillars in air on square array with lattice constant $a=0.54 \mu \mathrm{m}$ is presented. The radius and refractive index of rods are taken as $r=0.18 a$ and $n=3.4(\mathrm{Si})$, respectively. The perfect PC structure has a photonic bandgap for transverse electric modes, which extends from $\lambda=1.2 \mu \mathrm{m}$ to $1.7 \mu \mathrm{m}$ by using the plane wave expansion (PWE) calculation [21]. The $\lambda$ is the wavelength in free space. The channel drop filter consists of two PC waveguides and a microcavity is infiltrated with LCs, as shown in Figure 1. The ordinary and extraordinary refractive indices of the liquid crystal (5CB type) are $n_{\mathrm{LC}}^{o}=1.522$ and $n_{\mathrm{LC}}^{e}=1.706$, respectively. The diagram in Figure 1 indicates the director $n$ of a liquid crystal and the rotation angle $\phi$ of the director to the $z$-axis. The mesogenic temperature range of a single LC substance is usually quite limited [19]. For example, 5CB melts at $24^{\circ} \mathrm{C}$ and clears at $35.3^{\circ} \mathrm{C} .5 \mathrm{CB}$ is a nice material to work with because it exhibits a nematic phase at room temperature and its nematic range is more than 10 degrees. We assume that the operating temperature is at a constant room temperature and that the absorption loss is negligible.

The FDTD method is used to solve the light propagation in the 2D PC channel drop filter with LCs. The microcavity is used to select a single frequency light wave from a pulse, which propagates through the main waveguide and reroute it to another waveguide. The $Q$-factor and resonance frequencies of a PC microcavity are investigated. The $Q$-factor is defined as

$$
Q=\frac{\lambda_{0}}{\Delta \lambda},
$$

where $\lambda_{0}$ is the center wavelength and $\Delta \lambda$ is the full width at half power of the cavity's response.

We calculate the resonance frequency and $Q$-factor of the microcavity. The incident broadband source is introduced in the horizontal waveguide in Figure 1 and is separated from the microcavity. If both two PC waveguides and microcavity are infiltrated with LCs, the resonance frequency of the cavity is calculated to be $\lambda_{0}=1.502 \mu \mathrm{m}$ at $\phi=0^{\circ}$ and the $Q$-factor is 187.7 . If only microcavity is filled with LCs, the resonance frequency of the cavity is $1.435 \mu \mathrm{m}$ at $\phi=0^{\circ}$, and the $Q$-factor is 717.5. Therefore selectively infiltrating LCs results in a much higher $Q$-factor. A pulse of center wavelength $\lambda_{0}=1.435 \mu \mathrm{m}$ and of width $0.5 \mu \mathrm{m}$ was transmitted through the waveguide, which excited a single mode of oscillation inside the microcavity. The frequency responses obtained by taking the Fourier transform of the time-dependent field at the detecting points 2 and 3 are presented in Figure 2. It is transmission. In Figure 2(b) we can see that a peak frequency response at detecting point 3 occurs with the center wavelength $\lambda_{0}=1.435 \mu \mathrm{m}$ and spectral line width $\Delta \lambda=2 \mathrm{~nm}$. In the meantime, the detected frequency response at detecting point 2 is shown to have only very small amplitude at $\lambda_{0}=$ $1.435 \mu \mathrm{m}$ in Figure 2(c). Figure 3 shows that the resonance frequency of the cavity is shifted to $1.539 \mu \mathrm{m}$ at $\phi=90^{\circ}$, and the $Q$-factor is 769.5. The computer simulation of the

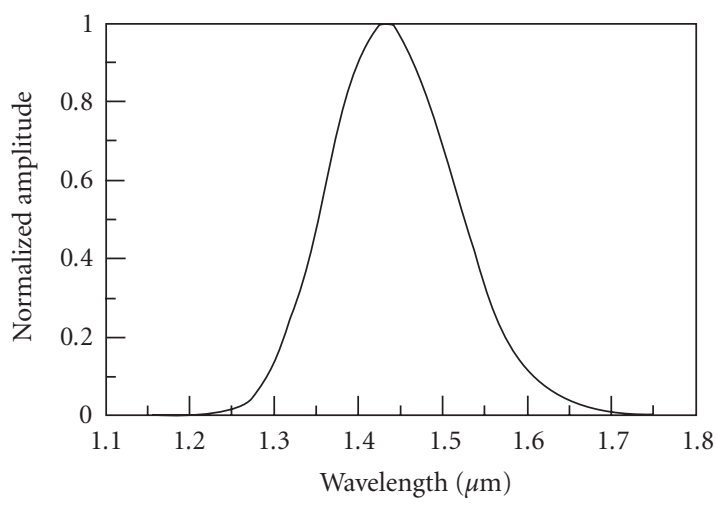

(a) An incident pulse of center wavelength $\lambda_{0}=1.435 \mu \mathrm{m}$ and of width $0.5 \mu \mathrm{m}$

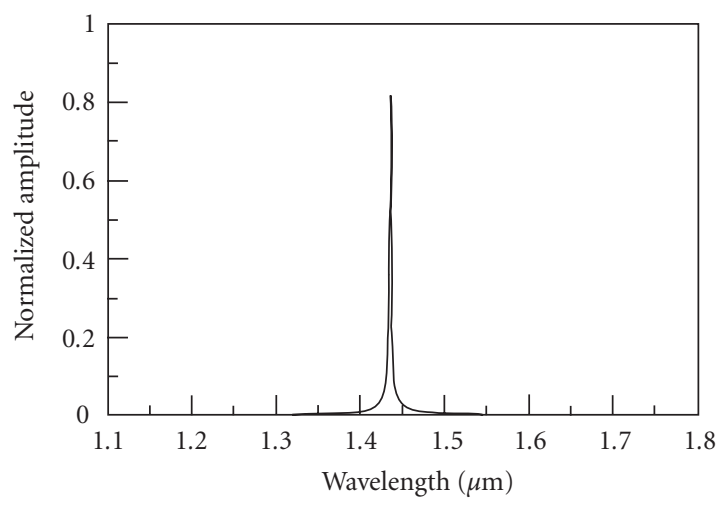

(b) The pulse of center wavelength $\lambda_{0}=1.435 \mu \mathrm{m}$ and $\Delta \lambda=2 \mathrm{~nm}$ measured at the detecting point 3

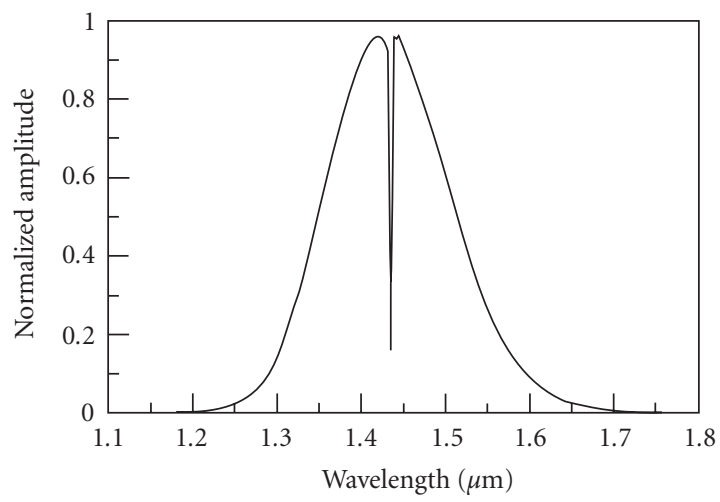

(c) The pulse measured at the detecting point 2

FIGURE 2: Wavelength spectrum of the incident pulse and the pulses measured at the detecting point.

resonant center wavelength as a function of rotation angle $\phi$ is presented in Figure 4. This means that for the broadband incident source we are able to achieve nearly 52 different channels by fine-tuning the refractive indices of LCs in the cavity. An example of multichannel design is discussed in Section 4.

In nematic LCs the directors of the LCs depend on the direction of the electric field in 2D planes. Indium tin oxide 


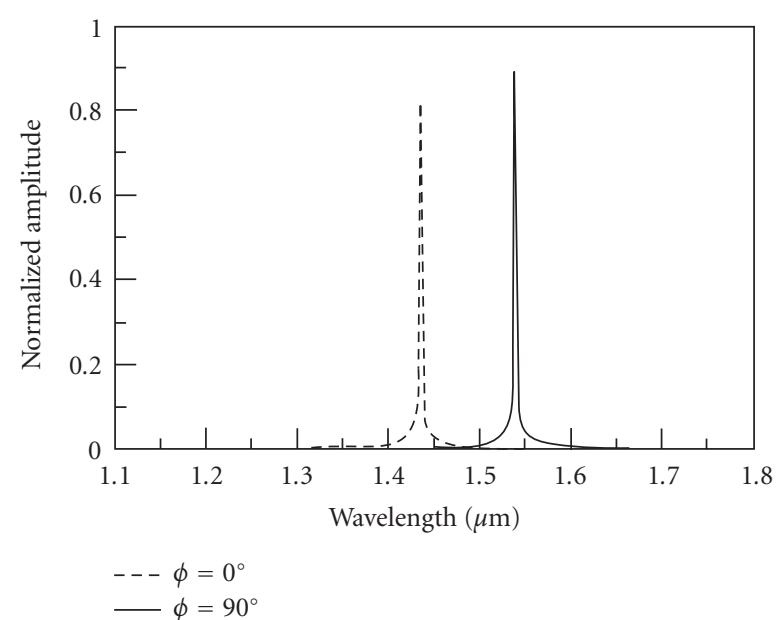

(a) The pulse of center wavelength $\lambda_{0}=1.539 \mu \mathrm{m}$ and $\Delta \lambda=$ $2 \mathrm{~nm}$ measured at the detecting point 3

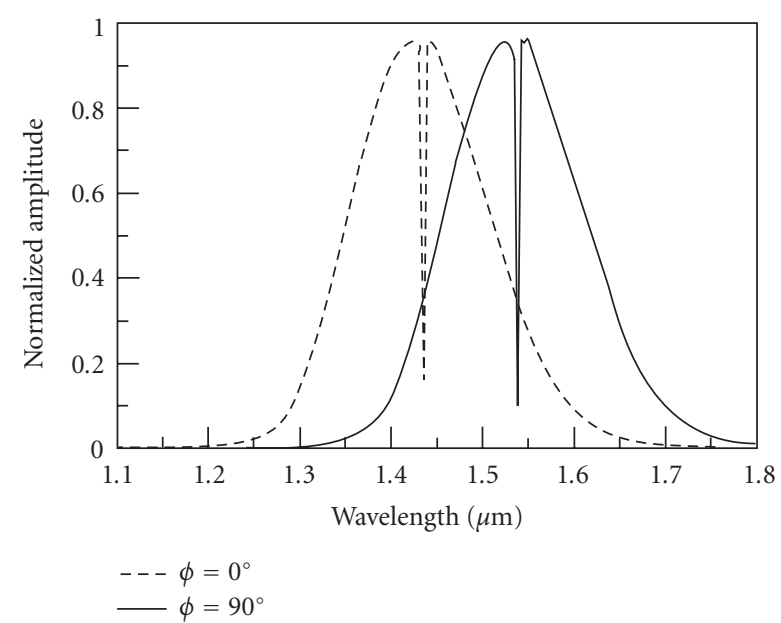

(b) The pulse measured at the detecting point 2

Figure 3: Wavelength shift in channel drop filter due to index modulation of liquid crystals.

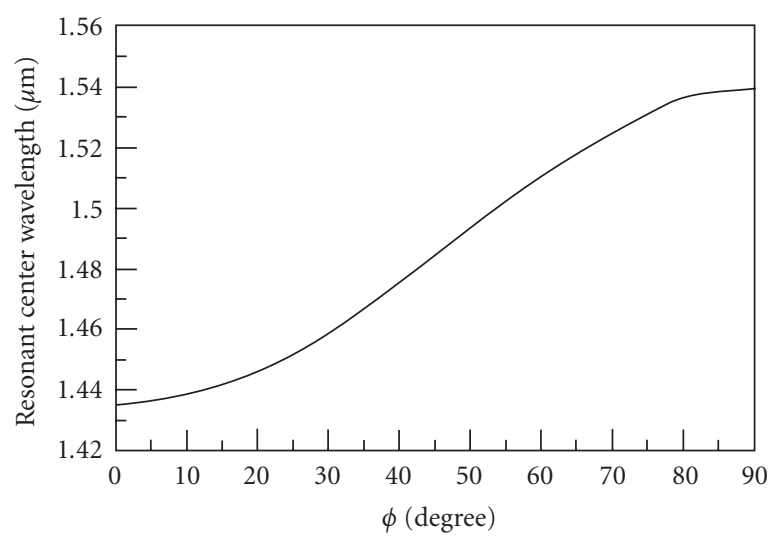

FIGURE 4: Calculated resonant center wavelength as a function of rotation angle $\phi$.

(ITO) layers can be attached to the top and the bottom of the PC channel drop filter, as shown in Figure 1. A surfactant has been applied to the surfaces of the ITO in contact with the liquid crystal, so the director tends to align itself in a single direction parallel to the flat surfaces of ITO. Let this be the $z$-axis. An electric field is applied perpendicular to the $z$-axis and parallel to the flat surfaces of the ITO. Let this be the $x$-axis. If the anisotropy of the dielectric susceptibility is positive, then the director tends to align along the electric field, rotating away from the $z$-axis toward the $x$-axis. Figure 5 shows the computer simulations of the rotation angle $\phi$ as a function of normalized voltage. The director can be reoriented by an electric field, when the field strength exceeds the Fréedericksz transition threshold [19]. When the applied voltage $V$ exceeds the Fréedericksz transition threshold ( $V$ th), the directors begin to tilt. $V$ th is the threshold

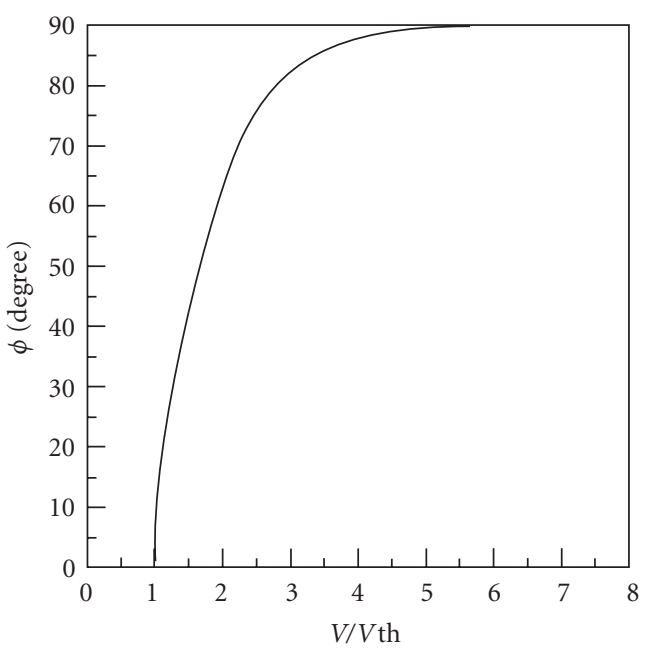

FIGURE 5: Calculated rotation angle $\phi$ as a function of normalized voltage. $V$ th is the threshold voltage.

voltage that is found to be $0.699 V_{\text {rms }}$ at $1 \mathrm{kHz}$ sinusoidal frequency. At $V<V$ th, the LC directors remain undisturbed and no phase change occurs. At $V>V$ th and in the small voltage region, the phase difference is linear to $V$ and the slope is determined by the ratio of refractive indices, elastic constants, dielectric constants, LC thickness, and wavelength. In general, the response time of an LC is of the order of a millisecond. However, it has been reported that the response time of LCs in nanoscale voids becomes of the order of $100 \mu \mathrm{s}$ [22]. The orientational relaxation times calculated by the molecular dynamics formalism and the experimental data determined by nuclear magnetic resonance spectroscopy for the nematic phase of 5CB crystal at $300 \mathrm{~K}$ were 


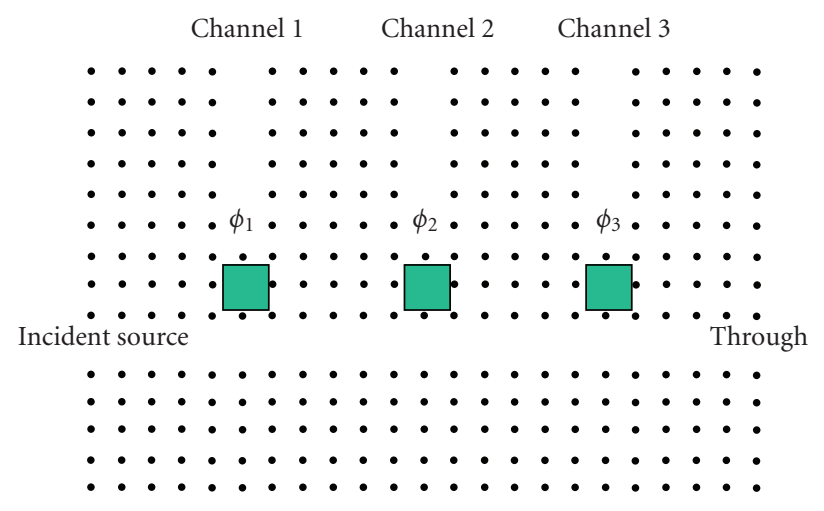

FIgURE 6: Multichannel wavelength division multiplexing that uses three microcavities with different rotation angle of liquid crystals.

presented in [23]. Therefore our novel PC waveguide channel drop filter with LCs can be used as either a fast optical router or a wavelength division multiplexing in photonic integrated circuits.

To understand how LCs respond to external fields, it must be realized that in most cases interactions between the LC molecules and boundaries have a large effect. These boundaries can be with a solid material such as glass, but the air-LC boundary is also both important and interesting. The orientation behavior of homogeneous planar layers of nematic LCs with open and closed ends in the field of compressional deformations caused by an acoustic effect is studied [24]. A free boundary value problem of nematic LCs with variable degree of orientation is also studied [25]. We are researching into the process of PC waveguide with localized LCs.

\section{MULTIPLECHANNEL WAVELENGTH DIVISION MULTIPLEXING}

A multichannel system with microcavities is considered. In the present studies, the system is chosen to be a three-channel system with three microcavities. The cavities are infiltrated with nematic LCs and the LCs in each cavity can have different rotational angles. Each channel is branched from the main waveguide, as shown in Figure 6. We assume that the rotation angle $\phi_{1}$ of channel 1 is $0^{\circ}$, the rotation angle $\phi_{2}$ of channel 2 is $45^{\circ}$, and the rotation angle $\phi_{3}$ of channel 3 is $90^{\circ}$. The propagations of the light wave in the three-channel system are calculated by the FDTD method. The wavelength spectrums obtained by taking the Fourier transform of the time-dependent field at each output port are presented in Figure 7. The Lorentzian line shapes can be seen in the figure. The center wavelength of each channel is seen to be directly proportional to the rotation angle of LCs. Each subchannel output different center frequency. The center wavelengths of subchannel are $\lambda_{1}=1.435 \mu \mathrm{m}$ at $\phi_{1}=0^{\circ}, \lambda_{2}=1.484 \mu \mathrm{m}$ at $\phi_{2}=45^{\circ}$, and $\lambda_{3}=1.539 \mu \mathrm{m}$ at $\phi_{3}=90^{\circ}$, respectively. The wavelength division multiplexing can be achieved by using tunable channel drop filter with nematic LCs. Further works

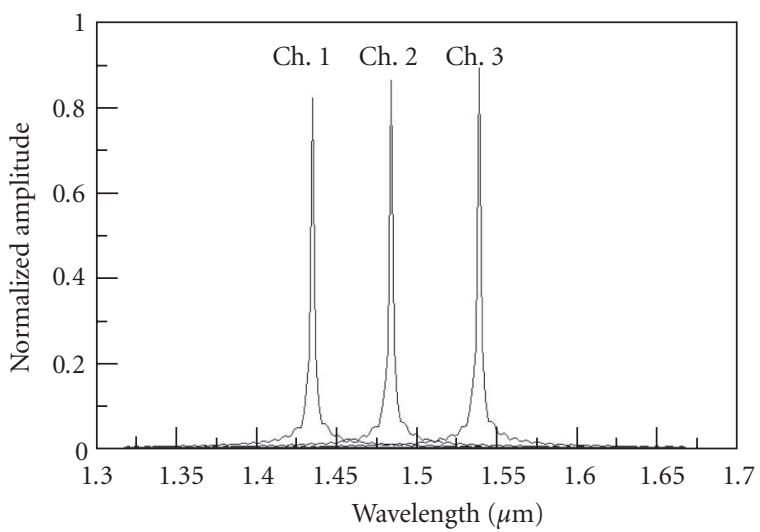

Figure 7: Wavelength spectrum of the pulses measured at each of the different channels of the multiple-channel wavelength division multiplexing.

will be on the multiple-channel WDM with an optimal array of higher $Q$-factor microcavities that can be used in an optical communication system.

\section{CONCLUSION}

In this paper we have demonstrated numerically a versatile photonic crystal modulated device by liquid crystal infiltrated cavities. A novel tunable PC channel drop filter infiltrated with nematic LCs was proposed and its properties were theoretically investigated by using the finite-difference time-domain method. The refractive indices of nematic LCs in the microcavity of 2D PC waveguides can be actively modulated by an electric field. Then we can control light propagation in PC waveguides. The effects of the indices of the LC on the $Q$-factors and resonance frequencies of a tunable PC channel drop filter are shown to be significant. The filter achieved spectrally separated channels with Lorentzian linewidths as small as $2 \mathrm{~nm}$. Therefore our tunable 2D PC channel drop filter modulated by nematic LC can be used to design a wavelength division multiplexing system. These results may facilitate novel applications of WDM devices in photonic integrated circuits.

\section{ACKNOWLEDGMENT}

The partial support by the National Science Council of Taiwan, Taiwan under Grant NSC94-2212-E-006-026 is gratefully acknowledged.

\section{REFERENCES}

[1] E. Yablonovitch, "Inhibited spontaneous emission in solidstate physics and electronics," Physical Review Letters, vol. 58, no. 20, pp. 2059-2062, 1987.

[2] J. D. Joannopoulos, R. D. Meade, and J. N. Winn, Photonic Crystals: Molding the Flow of Light, Princeton University Press, Princeton, NJ, USA, 1995.

[3] E. Centeno and D. Felbacq, "Guiding waves with photonic crystals," Optics Communications, vol. 160, no. 1-3, pp. 57-60, 1999. 
[4] R. D. Meade, A. Devenyi, J. D. Joannopoulos, O. L. Alerhand, D. A. Smith, and K. Kash, "Novel applications of photonic band gap materials: low-loss bends and high Q cavities," Journal of Applied Physics, vol. 75, no. 9, pp. 4753-4755, 1994.

[5] T. Baba, N. Fukaya, and J. Yonekura, "Observation of light propagation in photonic crystal optical waveguides with bends," Electronics Letters, vol. 35, no. 8, pp. 654-655, 1999.

[6] P. R. Villeneuve, D. S. Abrams, S. Fan, and J. D. Joannopoulos, "Single-mode waveguide microcavity for fast optical switching," Optics Letters, vol. 21, no. 24, pp. 2017-2019, 1996.

[7] P. R. Villeneuve, S. Fan, and J. D. Joannopoulos, "Microcavities in photonic crystals: mode symmetry, tunability, and coupling efficiency," Physical Review B, vol. 54, no. 11, pp. 7837-7842, 1996.

[8] S. Fan, S. G. Johnson, J. D. Joannopoulos, C. Manolatou, and H. A. Haus, "Waveguide branches in photonic crystals," Journal of the Optical Society of America B, vol. 18, no. 2, pp. 162$165,2001$.

[9] R. Costa, A. Melloni, and M. Martinelli, "Bandpass resonant filters in photonic-crystal waveguides," IEEE Photonics Technology Letters, vol. 15, no. 3, pp. 401-403, 2003.

[10] B.-K. Min, J.-E. Kim, and H.-Y. Park, "Channel drop filters using resonant tunneling processes in two-dimensional triangular lattice photonic crystal slabs," Optics Communications, vol. 237, no. 1-3, pp. 59-63, 2004.

[11] A. Sharkawy, S. Shi, and D. W. Prather, "Multichannel wavelength division multiplexing with photonic crystals," Applied Optics, vol. 40, no. 14, pp. 2247-2252, 2001.

[12] K. Busch and S. John, "Liquid-crystal photonic-band-gap materials: the tunable electromagnetic vacuum," Physical Review Letters, vol. 83, no. 5, pp. 967-970, 1999.

[13] K. Yoshino, Y. Shimoda, Y. Kawagishi, K. Nakayama, and M. Ozaki, "Temperature tuning of the stop band in transmission spectra of liquid-crystal infiltrated synthetic opal as tunable photonic crystal," Applied Physics Letters, vol. 75, no. 7, pp. 932-934, 1999.

[14] H. Takeda and K. Yoshino, "Tunable photonic band schemes of opals and inverse opals infiltrated with liquid crystals," Journal of Applied Physics, vol. 92, no. 10, pp. 5658-5662, 2002.

[15] H. Takeda and K. Yoshino, "Tunable light propagation in Y-shaped waveguides in two-dimensional photonic crystals utilizing liquid crystals as linear defects," Physical Review B, vol. 67, no. 7, 073106, 2003.

[16] H. Takeda and K. Yoshino, "Tunable light propagation in Y-shaped waveguides in two-dimensional photonic crystals composed of semiconductors depending on temperature," Optics Communications, vol. 219, no. 1-6, pp. 177-182, 2003.

[17] C.-Y. Liu and L.-W. Chen, "Tunable photonic crystal waveguide coupler with nematic liquid crystals," IEEE Photonics Technology Letters, vol. 16, no. 8, pp. 1849-1851, 2004.

[18] A. Taflove and S. C. Hagness, Computational Electrodynamics: The Finite Difference Time Domain Method, Artech House, Boston, Mass, USA, 1998.

[19] I.-C. Khoo and S.-T. Wu, Optics and Nonlinear Optics of Liquid Crystals, World Scientific, Singapore, 1993.

[20] M. Koshiba, Y. Tsuji, and S. Sasaki, "High-performance absorbing boundary conditions for photonic crystal waveguide simulations," IEEE Microwave and Wireless Components Letters, vol. 11, no. 4, pp. 152-154, 2001.

[21] H. Benisty, C. Weisbuch, D. Labilloy, et al., "Optical and confinement properties of two-dimensional photonic crystals,"
Journal of Lightwave Technology, vol. 17, no. 11, pp. 20632077, 1999.

[22] Y. Shimoda, M. Ozaki, and K. Yoshino, "Electric field tuning of a stop band in a reflection spectrum of synthetic opal infiltrated with nematic liquid crystal," Applied Physics Letters, vol. 79, no. 22, pp. 3627-3629, 2001.

[23] A. V. Zakharov and L. V. Mirantsev, "Dynamic and dielectric properties of liquid crystals," Physics of the Solid State, vol. 45, no. 1, pp. 183-188, 2003.

[24] O. A. Kapustina, "Orientation phenomena in nematic liquid crystals under a periodic compressional deformation," Acoustical Physics, vol. 50, no. 4, pp. 427-433, 2004.

[25] T. Isobe, "A free boundary value problem of nematic liquid crystals with variable degree of orientation," Nonlinear Analysis. Theory, Methods \& Applications, vol. 26, no. 2, pp. 149-169, 1996. 

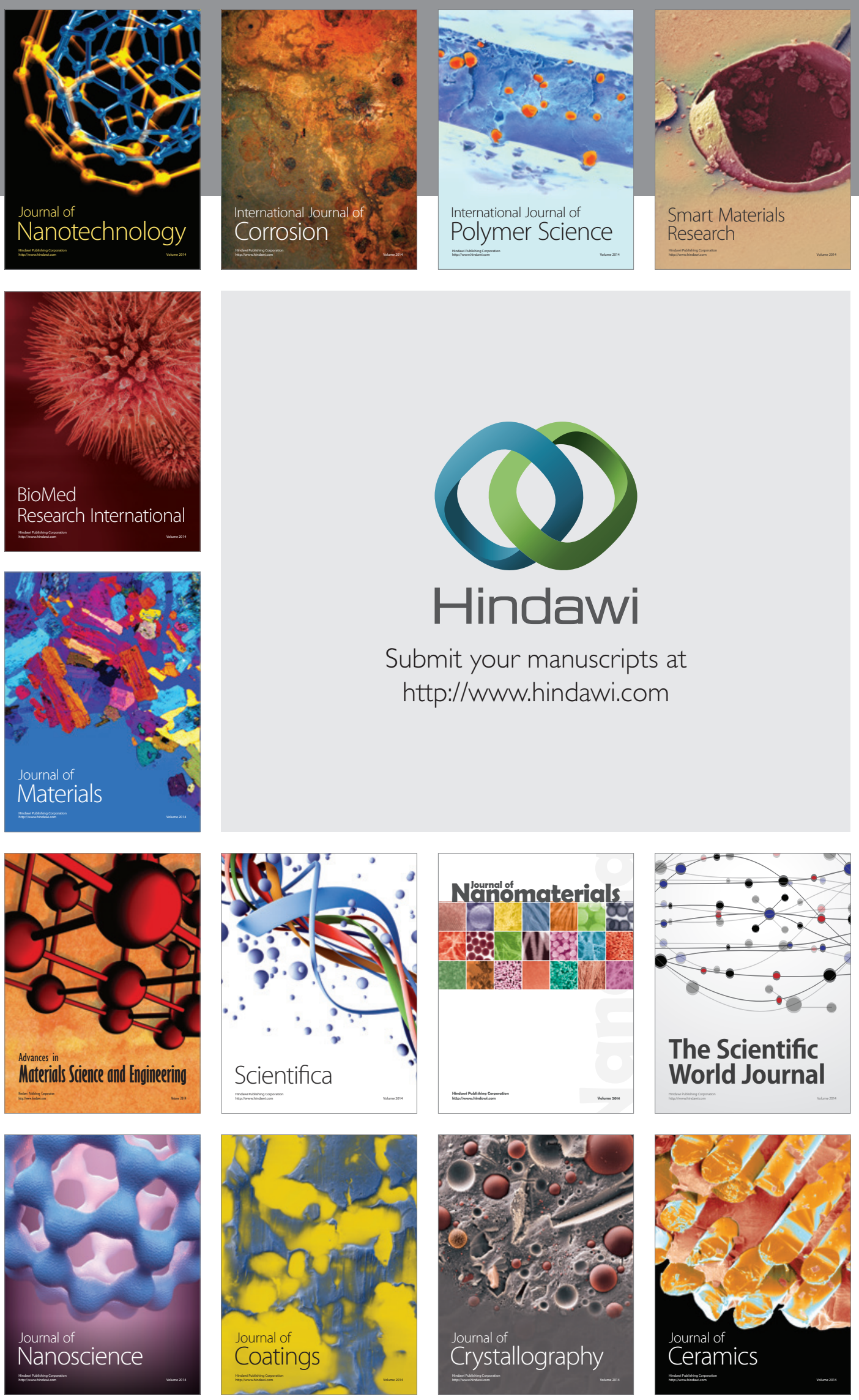

The Scientific World Journal

Submit your manuscripts at

http://www.hindawi.com

\section{World Journal}

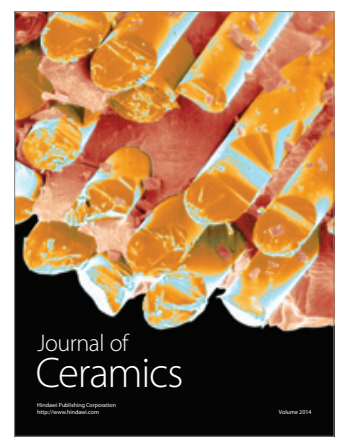

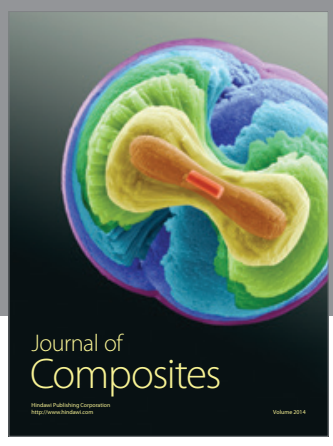
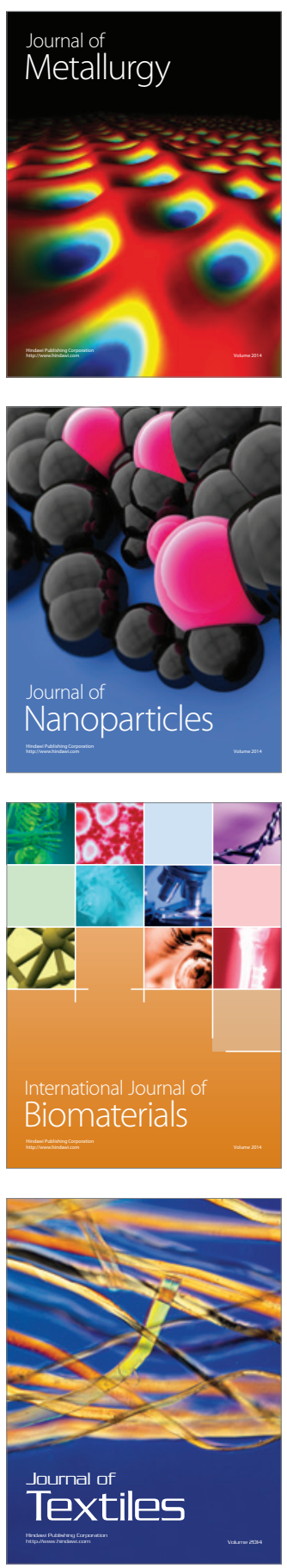